\title{
Diagnóstico postquirúrgico de Carcinoma Acinar gigante de glándula sublingual: Caso clínico
}

\author{
Post-surgical diagnosis of giant acinar carcinoma of the sublingual gland: Case report
}

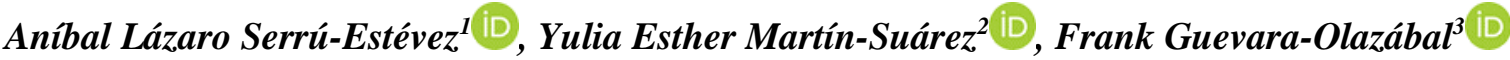 \\ 1 Especialista en 1er Grado en Oncología y Medicina General Integral. Profesor Instructor. Universidad de Ciencias \\ Médicas de Camagüey “Carlos Juan Finlay”, Hospital Docente de Oncología "María Curie”, Servicio de Cirugía de \\ Cabeza y Cuello \\ 2 Especialista en 1er Grado en Oncología y Medicina General Integral. Profesora Instructora. Universidad de Ciencias \\ Médicas de Camagüey “Carlos Juan Finlay”, Hospital Docente de Oncología "María Curie”, Servicio de Mastología. \\ 3 Especialista en 1er Grado en Cirugía Maxilofacial. Hospital "Amalia Simoni Argilagos”. Camagüey. Cuba. \\ Autor para la correspondencia. fguevaraolazabal@gmail.com \\ Fecha de recepción: 2 de abril de 2020 - Fecha de aceptación: 19 de abril de 2020
}

\section{RESUMEN}

El Carcinoma de Células Acinares de glándulas salivales es un tumor de bajo grado de malignidad que constituye entre el 3 17\% de los tumores malignos de glándulas salivales en general. Su aparición es más frecuente en la glándula parótida, pero es extremadamente rara en la glándula sublingual y aún más con ránula cervical siendo reportados en la literatura hasta el momento solo muy pocos casos a nivel mundial en dicha localización. El objetivo fue describir una paciente anciana enferma con una tumoración sublingual y cervical agresiva con un diagnóstico postquirúrgico definitivo de Carcinoma Acinar gigante de glándula sublingual con ránula cervical. El caso trata de una paciente femenina, de 87 años de edad, blanca, con antecedentes personales de mastectomía radical por Carcinoma Ductal de la mama izquierda, sin antecedentes patológicos familiares y sin hábitos tóxicos con historia clínica de haber sido atendida durante varios meses en centros no especializados por tumoración sublingual con empeoramiento clínico progresivo y divergencias diagnósticas relevantes. La paciente fue diagnosticada y tratada quirúrgicamente y se recuperó exitosamente, no obstante, el retraso en el diagnóstico y tratamiento certeros de los tumores malignos de glándulas salivales causados por la atención inicial de los pacientes en instituciones hospitalarias no especializadas en el tratamiento de las mismas debe ser fortalecido en vista a disminuir el tiempo y evitar tratamientos inadecuados por errores diagnósticos.

Palabras clave: Diagnóstico postquirúrgico, carcinoma acinar, glándula sublingual, ránula cervical.

\begin{abstract}
Salivary gland acinar cell carcinoma is a tumor with a low grade of malignancy that makes up between 3-17\% of tumors of salivary glands in general. Its appearance is more frequent in the parotid gland, but it is extremely rare in the sublingual gland and even more with cervical ranula, being reported in the literature so far only very few cases worldwide with such location. The goal was to describe a sick elderly patient with an aggressive sublingual and cervical tumor with a definitive post-surgical diagnosis of giant acinar carcinoma of sublingual gland with cervical ranula. The case involves a white female patient, 87year-old, with a personal history of radical mastectomy by Ductal Carcinoma of the left breast, no family pathological history and no toxic habits with a medical history of having been attended for several months in non-specialized centers by a sublingual tumor with progressive clinical worsening and relevant diagnostic divergences. The patient was diagnosed and treated surgically and successfully recovered, however, the delay in the accurate diagnosis and treatment in malignant salivary gland tumors caused by the initial care of patients in hospital institutions non-specialized in the treatment of salivary glands should be strengthened in view of decreasing time and avoiding inadequate treatments by diagnostic errors.
\end{abstract}

$\underline{\text { Keywords: }}$ Post-surgical diagnosis, acinar carcinoma, sublingual gland, cervical ranula.

\section{INTRODUCCIÓN}

Los tumores de las glándulas salivales mayores y menores se caracterizan por ser de variado comportamiento biológico y poseer diferentes tipos y subtipos histopatológicos, (Costa, Altemani, \& Hermsen, 2011;
Nagao, 2013; Valstar et al., 2017; Hukkanen, Dintzis, \& Treuting, 2017; Kessler \& Bhatt, 2018) lo cual combinado con su baja incidencia hace difícil evaluar su historia natural, factores, pronósticos y resultados logrados con las distintas modalidades de tratamiento, de una manera 
prospectiva y aleatorizada. (Arrangoiz et al., 2013; Marteletti et al., 2017). Las neoplasias de glándulas salivales son poco frecuentes, y corresponden según la literatura a 3-6\% de los tumores de cabeza y cuello, y a $0.6 \%$ de todas las neoplasias. Tienen una incidencia global estimada en 0.4-13.5 por cada 100,000 personas anualmente y estas incluyen un grupo histopatológico heterogéneo que va desde neoplasias benignas hasta neoplasias malignas muy agresivas de mal pronóstico y con comportamientos clínicos y manejo terapéutico totalmente distintos (Shah, Patel, Singh, \& Wong, 2012; Tian, Wang, Hu, \& Li, 2010; Neville, Damm, Chi, \& Allen, 2015; Campolo et al., 2018).

El carcinoma de células acinares era denominado antiguamente adenoma de células serosas o simplemente adenoma salival y fue considerado una lesión benigna hasta 1953, año en el cual se le atribuyó un potencial maligno y puede aparecer en diferentes partes como el duodeno, los senos, las glándulas salivales (Ilayaraja, Prasad, Anuthama, \& Sruthi, 2014; Munteanu et al., 2012; Hamidian et al., 2013; Limite et al., 2014; Avila et al., 2019). Hoy en día debido a su potencial de recurrencia, metástasis y hasta de muerte la Organización Mundial de Salud (OMS) lo reconoce como un carcinoma maligno de bajo grado (Castro \& Reyes, 2016; Caliskan, Nurdan, \& Henderson, 2015).

$\mathrm{Su}$ incidencia varía según distintos autores, algunos refieren que representa el 1 al 6\% según Caliskan et al. (2015) de los tumores malignos de las glándulas salivales, y otros autores reportan que constituye del 10 al $12 \%$ de todas las neoplasias malignas de glándulas salivales siendo la parótida las más afectada (Andreoli, Andreoli, Shrime, \& Devaiah, 2012; Scherl et al., 2018; Lewis, Tong, \& Maghami, 2016). Se reporta además que los tumores de la glándula sublingual son raros y en su mayoría son malignos, constituyendo su diagnóstico un reto para todo cirujano de cabeza y cuello (Adirajaiah, Anehosur, Sumana, \& Gopalakrishnan, 2012; Sun et al., 2010). Este tumor maligno representa el tercero más común en la edad adulta detrás del carcinoma mucoepidermoide (Zambrano, López, Harris, Reinhard, \& Redondo. 2018; Castro \& Reyes 2016) y del carcinoma adenoideo quístico; en la edad pediátrica es el segundo más frecuente por detrás del carcinoma mucoepidermoide. Es un poco más frecuente en el sexo femenino y la edad a la que se presentan es menor a la observada en otros tumores malignos (Lee, 2012). Este tumor de bajo grado tiende a recurrir, aunque lo atribuyen a cuando la resección no es completa. Las metástasis distantes ocurren hasta en un $13 \%$ de los pacientes y los sitios de metástasis más frecuentes son los ganglios linfáticos cervicales, el hígado, los pulmones, huesos (mayormente columna dorsal), cerebro y órbita contralateral (Escajadillo, 2014; Harrison, 2014).

La ránula intraoral es una enfermedad benigna seudoquística de extravasación que tiene su origen en las glándulas salivales sublinguales (Guzmán-Letelier, Crisosto-Jara, Reyes-Marislao, Peñarrocha-Diago, \& Peñarrocha-Oltra, 2016; Flaitz, 2018) cuando están presentes pueden complicar los casos de carcinomas de células acinares de las glándulas sublinguales, las ránulas cervicales son entidades muy poco frecuentes (Choi, Bae, Kim, \& Song, 2015; Sharma, Singhal, \& Ragesh, 2016) y deben ser tratados con la mayor anticipación, en este trabajo se presenta un caso en el cual esto no se cumplió, aunque posteriormente fue resuelto con éxito en una paciente octogenaria.

El objetivo de esto estudio fue describir una paciente anciana enferma con una tumoración sublingual y cervical agresiva con un diagnóstico postquirúrgico definitivo de carcinoma acinar gigante de glándula sublingual con ránula cervical.

\section{REPORTE DEL CASO}

Se trata de una paciente femenina de 87 años procedente del municipio Ciro Redondo, provincia Ciego de Ávila, Cuba, viuda, jubilada, con antecedentes de haber recibido tratamiento quirúrgico hace un año de Mastectomía radical por Carcinoma Ductal de la mama izquierda, sin antecedentes patológicos familiares y sin hábitos tóxicos. La misma acudió al Hospital Provincial de Ciego de Ávila en octubre del 2017 por aumento de volumen bajo la lengua que le molestaba para la alimentación regular. Fue evaluada por el servicio de Maxilofacial y se le realizó una biopsia inicial que informó: Biopsia (B-2446-17): Ránula. Posteriormente y ante el incremento de tamaño de la tumoración se le realizó una segunda biopsia que informó: Biopsia (B-17-2910): Ameloblastoma común, patrón plexiforme.

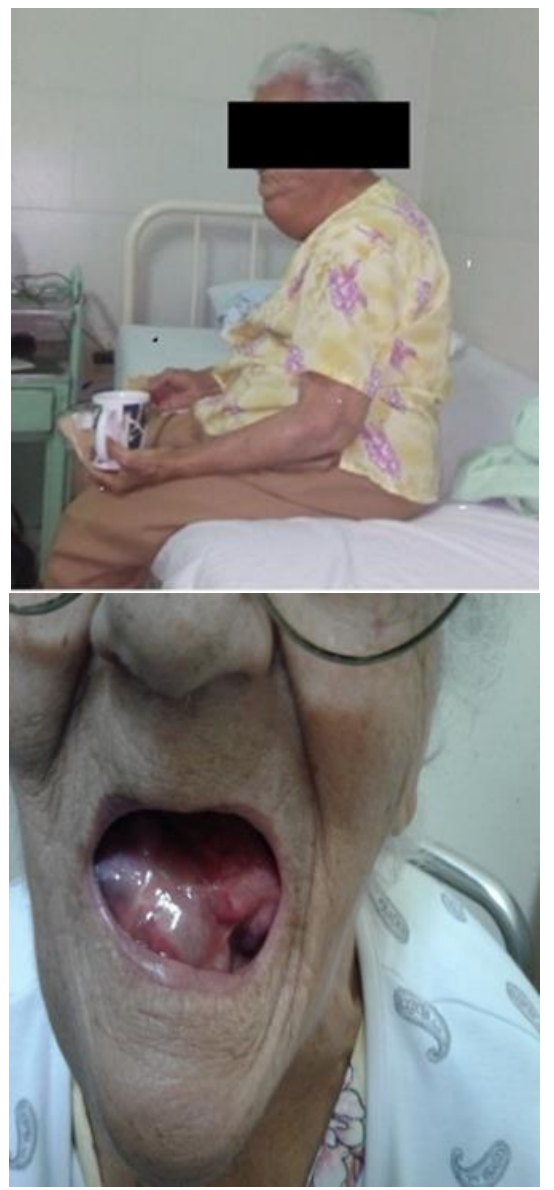

Imágenes 1 y 2. Paciente a la inscripción al centro médico. 
Ante la divergencia diagnóstica es enviada al Hospital Oncológico María Curie de Camagüey donde es atendida por el Servicio de Cirugía de Cabeza y Cuello. Para ese momento presentaba por debajo de la lengua y lateralizada a la derecha tumoración violácea, de consistencia blanda, renitente, aparentemente no adherida a planos profundos y que impresionaba tener algún componente vascular incluido, esto dificultaba el habla y la deglución, de aproximadamente $3 \mathrm{~cm}$ de diámetro y otra más pequeña de $0.5 \times 0.5 \mathrm{~cm}$ de diámetro, levemente posterior a la antes descrita. Se encontró además masa cervical derecha de un mes de evolución y crecimiento progresivo que había alcanzado aproximadamente $6 \times 4 \mathrm{~cm}$ de diámetro, de consistencia blanda y ligeramente renitente, que involucraba desde el nivel I hasta el nivel III.

Se decide repetir biopsia por aspiración por aguja fina (BAAF), en ese momento y la misma informó: Biopsia (B17-2910): Sialoadenoma papilífero o papilar de glándula salivar menor que puede recidivar. Se decide realizar exéresis de la tumoración y se lleva a cirugía el 19 de marzo del 2018, fecha en la cual la tumoración cervical había incrementado su volumen hasta casi ocupar el hemicuello derecho. Posteriormente, se decidió realizar abordaje cervical inicial y se encuentra una tumoración de consistencia irregular que se extiende desde el nivel I al IV adherida a los grandes vasos de cuello y a las estructuras nerviosas locales por elementos fibrosos que dificultaban el abordaje. Se realiza exéresis ascendente de la lesión logrando su separación de las estructuras cervicales hasta llegar al nivel I (submentoniano) sitio donde trasponía la musculatura del suelo de la boca la cual se atraviesa y se realiza exéresis de la lesión glandular sublingual principal. Por lo extendido del tiempo quirúrgico (7 horas) y el estado general de la paciente se decide postergar el abordaje de la lesión más pequeña para un segundo tiempo quirúrgico el cual se llevó a cabo el 14 de mayo del 2018 con resultados satisfactorios.

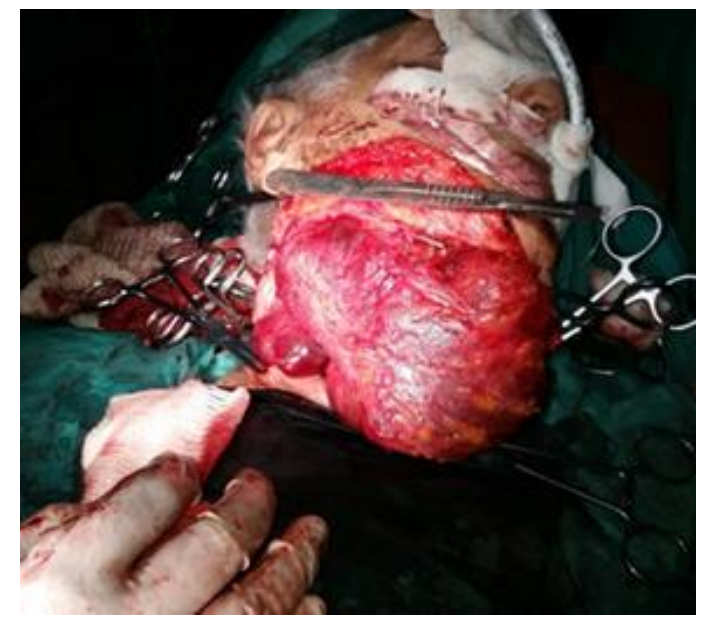

Imagen 3. Paciente en el transoperatorio.

La biopsia de la primera cirugía informó: Biopsia (B-64518): Tumor epitelial primario maligno de bajo grado tipo carcinoma acinar variante quístico papilar; y la biopsia de la segunda intervención quirúrgica informó: Biopsia (B1115-18): Adenocarcinoma acinar quístico papilar primario de glándula salival bien resecado.

La evolución posterior fue satisfactoria y sin complicaciones. Dada la ausencia de adenopatías cervicales y ante la negativa familiar de recibir radioterapia posterior dado el buen estado general de la paciente y su edad se concluye con seguimiento estrecho por parte de nuestro servicio y el diagnóstico definitivo de carcinoma acinar de glándula sublingual variante quístico papilar.

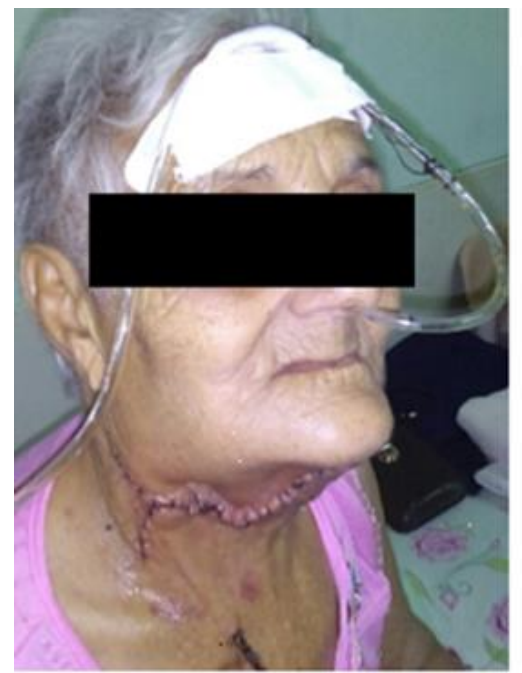

Imagen 4. Paciente en el postoperatorio.

\section{DISCUSIÓN}

El diagnóstico de los tumores malignos de glándulas es complejo por lo que frecuentemente, los enfermos son derivados de un centro no especializado, no sin antes habérseles practicado la resección parcial o total de un tumor primario insospechado de maligno o simplemente con una biopsia quirúrgica en un sitio inapropiado. Aún hoy, no más del $6 \%$ de los enfermos es derivado con el resultado citológico de una punción con aguja fina. Por otro lado, existen algunas razones que retrasan la sospecha de un cáncer. A su baja frecuencia relativa con los tumores benignos, los procesos inflamatorios, litiásicos o metastásicos, se agrega un signo común al conjunto: la presencia de tumor (Marteletti et al., 2017). En nuestra paciente este fue un punto esencial al ser evaluada y posteriormente remitida desde un hospital no especializado en la atención de las afecciones malignas y sin un servicio constituido y especializado de Cirugía de Cabeza y Cuello lo cual dilató en el tiempo su diagnóstico y posterior tratamiento quirúrgico.

Las ránulas según Sharma et al. (2016) son por definición un mucocele en el piso de la lengua en relación a las glándulas sublinguales y aparecen usualmente como una tumoración fluctuante, renitente, a veces con un tinte azulado, mayores de $2 \mathrm{~cm}$ y que impresiona que se encuentra a tensión. El sitio más común es la región lateral del suelo de la boca. La ránula cervical (Plunging ránula) tiende a aparecer después de la tercera década de la vida y aparece cuando la presión del fluido logra abrirse paso a través de la perforación del músculo milohioideo al espacio submandibular (Sharma et al., 2016). El diagnóstico inicial de ránula sobre todo cuando se extendió a la región cervical era de inicio el más probable dado los elementos clínicos de la enfermedad, pero el 
crecimiento acelerado de la extensión cervical de menos de un mes podría hacer sospechar la etiología maligna de la lesión, aunque los carcinomas de células acinares tienden a tener un crecimiento lento.

Estas neoplasias se originan como resultado de la proliferación neoplásica o citodiferenciación aberrante de la reserva celular pluripotencial que se encuentra en la unión entre los acinos y los conductos intercalados glandulares (Escajadillo, 2014). En este caso el patrón encontrado fue el papilar quístico, se describen solo cuatro patrones de los cuales el sólido y el microquístico son los más frecuentes. El patrón papilar-quístico es menos frecuente que los anteriores, mientras que el folicular es el más raro (Rosero et al., 2011).

La aparición de un carcinoma de células acinares en la glándula sublingual es extremadamente raro, Choi et al. (2015) refieren que este tipo de tumor asociado a ránula cervical solo ha sido reportado en ocho ocasiones en la literatura mundial, siendo la última el caso reportado por este trabajo, el cual constituye la novena ocasión documentada en la literatura, lográndose con éxito la recuperación de la paciente que tiene una edad prolongada. Su tratamiento consiste esencialmente en la resección quirúrgica completa del tumor. La radioterapia está indicada si existen márgenes positivos, extensión extra glandular, preservación del nervio facial con márgenes próximos, invasión perineural, ganglios metastásicos, tumores de alto grado y tumores de bajo grado recurrentes. Todos estos representan a su vez riesgo de recurrencia (Escajadillo, 2014). Caliskan et al. (2015) reportaron un caso de parótida de 75 años y Andreoli et al. (2012) reportaron en esa misma glándula pacientes entre 5 y 95 años. Zambrano et al. (2018) trataron un carcinoma mucodermoide palatino en un joven de 18 años y Sharma et al. (2016) también reportaron un caso de la misma edad. Choi et al. (2015) insisten en el tratamiento a tiempo de estas patologías.

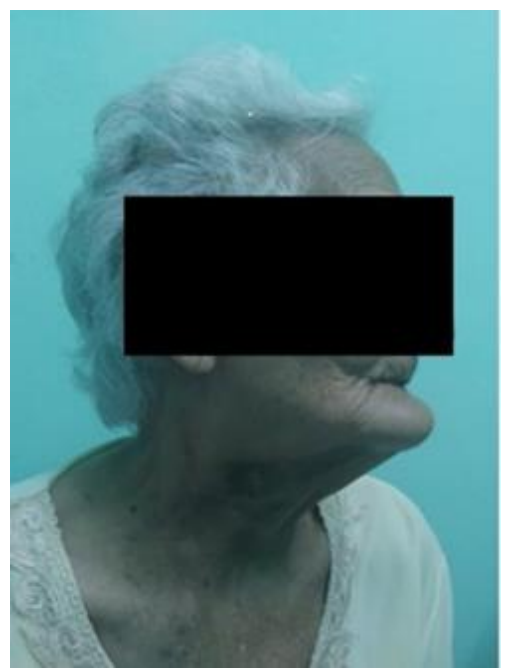

Imagen 5. Paciente 6 meses después de la cirugía.

\section{CONCLUSIONES}

El retraso en el diagnóstico y tratamiento de los tumores malignos de glándulas salivares causado por la atención inicial de los pacientes en instituciones hospitalarias no especializadas en el tratamiento de las mismas constituye un eslabón a fortalecer en vista disminuir el tiempo y evitar tratamientos inadecuados por errores diagnósticos. Es importante recordar que, aunque infrecuente a nivel de la glándula sublingual el carcinoma de células acinares existe, puede existir asociado a una ránula cervical y puede tratarse quirúrgicamente con éxito aún a edades avanzadas.

\section{REFERENCIAS}

Adirajaiah, S., Anehosur, V., Sumana, Gopalakrishnan, K. (2012). Adenocarcinoma of the sublingual salivary gland: A case report. Journal of Oral Biology and Craniofacial Research, 2(3), 206-209. https://doi.org/10.1016/j.jobcr.2012.10.004

Andreoli, M. T., Andreoli, S. M., Shrime, M. G., \& Devaiah, A. K. (2012). Radiotherapy in parotid acinic cell carcinoma: does it have an impact on survival? Archives of Otolaryngology - Head \& Neck Surgery, 138(5), 463-466. https://doi.org/10.1001/archoto.2012.226

Arrangoiz, R., Galloway, T., Lango, M., Ridge, J., Sarcu, D., Papavasiliu, P. (2013). Current thinking on malignant salivary gland neoplasms. Journal of Cancer Treatment and Research, 1(1), 8-24. https://doi.org/10.11648/j.jctr.20130101.12

Avila, R. E., Samar, M. E., Fonseca, I. B., Corball, A. G., Carriel, V., García-Martínez, L., \& Rodríguez, I. (2019). Proliferaciones Oncocíticas de Glándulas Salivales: Estudio Estructural e Inmunohistoquímico de 7 Casos. International journal of Odontostomatology, 13(1), 82-88. https://dx.doi.org/ 10.4067/S0718-381X2019000100082

Caliskan, B., Nurdan, K. A., \& Henderson, R. (2015). A rare and unusual case of acinic cell carcinoma of parotid gland evaluated by F-18 FDG PET/CT. Acta Medica Anatolia, 3(3), 105-107.

Campolo, A. G., Ramírez, H. S., Vargas, A. D., León, A. R., Goñi, I. E., \& Solar, A. G. (2018). Perfil epidemiológico de neoplasias epiteliales de glándulas salivales. Revista médica de Chile, 146(10), 1159-1165. http://dx.doi.org/10.4067/S003498872018001001159

Castro, M. C. C., \& Reyes, O. L. M. (2016). Carcinoma de células acinares. Reporte de caso y revisión de la literatura. Revista Médica Carrionica, 3(2), 138-143.

Costa, A. F., Altemani, A., \& Hermsen, M. (2011). Current concepts on dedifferentiation/high grade transformation in salivary gland tumors. Pathology Research International, 2011, Article ID 325965, 9 págs. https://doi.org/10.4061/2011/325965

Choi, Y. S., Bae, C. H., Kim, Y-D., \& Song, S-Y. (2015). A case of acinic cell carcinoma associated with ranula in sublingual gland. Korean Journal of Otorhinolaryngol-Head Neck Surgery, 58(9), 637-640. https://doi.org/10.3342/kjorlhns.2015.58.9.637

Escajadillo, J. R. (2014). Oídos, nariz, garganta y cirugía de cabeza y cuello. ( $4^{\text {th }}$ ed.). $974 \mathrm{pp}$. México: Editorial El Manual Moderno. 
Flaitz, C. (2018). What is the pathophysiology of mucocele and ranula? MEDSCAPE. Disponible en https://www.medscape.com/answers/1076717103413/what-are-the-differential-diagnoses-formucocele-and-ranula.

Guzmán-Letelier, M., Crisosto-Jara, C., Reyes-Marislao, B., Peñarrocha-Diago, M., \& Peñarrocha-Oltra, D. (2016). Marsupialización modificada de ránula gigante intraoral: reporte de caso. Revista Clínica Periodoncia Implantología y Rehabilitación Oral, 9(3), 217-221. https://doi.org/10.1016/ j.piro.2015.06.008

Hamidian, J. A., Shokouh-Amiri, H., Wellman, G., Hobley, J., Veluvolu, A., \& Zibari, G. B. (2013). Acinar cell carcinoma presenting as a duodenal mass: review of the literature and a case report. The Journal of the Louisiana State Medical Society, 165(1), 20-23, 25.

Hukkanen, R. R., Dintzis, S. M., \& Treuting, P. M. (2017). Salivary glands. (Chapter 8). In: Treuting, P. M., \& Dintzis, S. M. (Eds.). Comparative Anatomy and Histology (2nd ed.). 135-145 pp. Print Book \& EBook.

Harrison, L. B. (2014). Head and neck cancer: A multidisciplinary approach. ( $\left.4^{\text {th }} \mathrm{ed}\right) .960 \mathrm{pp}$. Wolters Kluwer/Lippincott Williams \& Wilkins.

Ilayaraja, V., Prasad, H., Anuthama, K., \& Sruthi, R. (2014). Acinic cell carcinoma of minor salivary gland showing features of high-grade transformation. Journal of Oral Maxillofacial Pathology, 18(1), 97101. http://dx.doi.org/10.4103/0973-029X.131925

Kessler, A. T., \& Bhatt, A. A. (2018). Review of the major and minor salivary glands, Part 2: Neoplasms and tumor-like lesions. Journal of Clinical Imaging Science, 8(1), 48. https://doi.org/10.4103/jcis.JCIS_46_18

Lee, K. J. (2012). Essential otolaryngology. Head and Neck Surgery. (10 ${ }^{\text {th }}$ ed.). Editorial McGraw-Hill, $1117 \mathrm{pp}$.

Lewis, A. G., Tong, T., \& Maghami, E. (2016). Diagnosis and management of malignant salivary gland tumors of the parotid gland. Otolaryngologic Clinics of North America, 49(2), 343-380. http://dx.doi.org/10.1016/j.otc.2015.11.001

Limite, G., Di Micco, R., Esposito, E., Sollazzo, V., Cervotti, M., Pettinato, G., Varone, V., Benassaia, G., Amatoa, B., Pilone, V., Luglio, G., Vitiello, A., Hasania, A., \& Liccardo, F. (2014). Acinic cell carcinoma of the breast: review of the literature. International Journal of Surgery, 12(1), S35-S39. https://doi.org/10.1016/j.ijsu.2014.05.004

Marteletti, C., Lermer, D., González Aguilar, G., Rubino, A., Delgado, D., Carrizo, G. (2017). Carcinoma de las glándulas salivales. Revista Argentina de Cirugía, 109(2), 68-72.
Munteanu, M. C., Margaritescu, C., Cionca, L., Nitulescu, N. C., Daguci, L., \& Ciuca, E. M. (2012) Acinic cell carcinoma of the salivary glands: A reterospective clinicopathologic study of 12 cases. Romanian Journal of Morphology and Embryology, 53(2), 313-320.

Nagao, T. (2013). Dedifferentiation and high-grade transformation in salivary gland carcinomas. Head and Neck Pathology, 7(1), S37-S47. https://doi.org/10.1007/s12105-013-0458-8

Neville, B. W., Damm, D. D., Chi, A. C., \& Allen, C. M. (2015). Oral and maxillofacial pathology. ( $4^{\text {th }} \mathrm{ed}$.), 928 pp. Elsevier.

Rosero, D. S., Álvarez, R. A., Sota, P. O., Puertas, A. C., Vicente, S. A., \& Mejía, E. U. (2011). Carcinoma de células acinares con tres variantes morfológicas. Cursos y Congresos/Reuniones Anuales SEAPIAP/XXXIV Reunión Anual SEAP 2011/Pósteres.

Shah, J. P., Patel, S. G., Singh, B., \& Wong, R. (2012). Jatin Shah's head and neck surgery and oncology. (5 ${ }^{\text {th }}$ ed.). 896 pp. Elsevier.

Sharma, S. K., Singhal, G., \& Ragesh, V. G. (2016). A cervical ranula: A case report. International Journal of Medical Research \& Health Sciences, 5(7), 109-113.

Scherl, C., Kato, M. G., Erkul, E., Graboyes, E. M., Nguyen, S. A., Chi, A. C., Morgan, P. F., Day, T. A. (2018). Outcomes and prognostic factors for parotid acinic cell carcinoma: A National Cancer Database study of 2362 cases. Oral Oncology, 82, 53-60. https://doi.org/10.1016/j.oraloncology.2018.05.002

Sun, G., Yang, X., Tang, E., Wen, J., Lu, M., \& Hu, Q. (2010). The treatment of sublingual gland tumors. International Journal of Oral Maxillofacial Surgery, 39(9), 863-868. https://doi.org/10.1016/j.ijom.2010.04.051

Tian, Z., Li, L., Wang, L., Hu, Y., \& Li, J. (2010). Salivary gland neoplasms in oral and maxillofacial regions: a 23-year retrospective study of 6982 cases in an eastern Chinese population. International Journal of Oral Maxillofacial Surgery, 39(3), 235242. https://doi.org/10.1016/j.ijom.2009.10.016

Valstar, M. H., de Ridder, M., van den Broek, E. C., Stuiver, M. M., van Dijk, B. A., van Velthuysen, M. L., Balm, A. J. M., \& Smeele, L. E. (2017). Salivary gland pleomorphic adenoma in the Netherlands: A nationwide observational study of primary tumor incidence, malignant transformation, recurrence, and risk factors for recurrence. Oral Oncology, 66, 93-99. https://doi.org/10.1016/j.oraloncology.2017.01.004

Zambrano, J., López, E., Harris, J., Reinhard, S., \& Redondo, C. (2018). Carcinoma mucoepidermoide palatino de bajo grado de malignidad en adolescente. Revista Ciencias y Salud Virtual, 10(2), 72-81. https://doi.org/https://doi.org/10.22519/21455333.11 41 